\title{
Linear Complexity of Generalized Hall's Sextic Sequence
}

\author{
Runping Ye \\ Education Department of Suqian College, Suqian, Jiangsu, P R China \\ e-mail: yeziping168@sina.com
}

\begin{abstract}
Let $p$ be a primes with $p \equiv 1(\bmod 6)$. In this paper,we calculate the linear complexity of the connection polynomial of a balanced binary Hall's sextic sequence. It shows that the sequence has large linear complexity and balanced properties.
\end{abstract}

Keywords-generalized cyclotomic sequence, autocorrelation value, linear complexity

\section{INTRODUCTION}

For cryptographic applications, pseudo-random sequences are required to have the property of unpredictability. Balance and linear complexity are two main components that indicate this feature. A lot of authors investigate the minimal polynomials and the linear complexity of cyclotomic sequences (see [2,4,5,6,7,9]). In 1997, Ding [2] calculated the linear complexity of generalized cyclotomic binary sequences of order 2. In 2001, Kim and Song [7] got the linear complexity of cyclotomic binary sequences of order 6. Recently, Edemskii [4] obtained partial exponential sums of cyclotomic classes of order 4 and 6 in an extension over $\mathrm{GF}(2)$ to compute the linear complexity of binary sequences and [5] gave a method to calculate the linear complexity of generalized cyclotomic binary sequences of period $p^{n}$.

Also some applications, such as communication systems and cryptography require a sequence is easy to distinguish from a time-shifted version of itself, which is to say: have low autocorrelation. So there are several authors investigate difference sets and low correlation values of binary sequences (see $[1,2,3,8]$ ). Liu et al. [10] computed the autocorrelation values and the linear complexity of $\mathrm{DH}$ generalized cyclotomic sequences of period $p^{n}$ over GF(q) provided that $p=q f+1$ and $q$ are primes, which generalized Sidelnikov's results [11].

A sequence $\left(S=s_{0}, S_{1}, \cdots, s_{N-1}, \cdots\right)$ is said to be $N$ periodic if $S_{i}=s_{i+N}$ for all $i \geq 0$. The linear complexity of a sequence $S$ over $\mathrm{GF}(2)$ is an important characteristic of its equality. It is defined to be the smallest positive integer $L$ for which there exist constants $C_{1}, \cdots, C_{L} \in \mathrm{GF}(2)$ such that

$$
S_{g}=c_{1} S_{g-1}+c_{2} S_{g-2}+\cdots+c_{L} S_{g-L} \text { for all } g \geq L .
$$

We call $f(x)=1-c_{1} x-\cdots c_{L} x^{L}$ the connection polynomial.

Let $p=6 f+1$ be a odd prime, where $f$ is a positive integer. Let $\theta$ be a primitive root of $p$ Set $D=\left(\theta^{6}\right)$ a cyclic subgroup of $Z_{p}^{*}$. There are six classes $D_{i}=\theta^{i} D$, $i=0,1,2,3,4,5$, and $Z_{p}^{*}=\bigcup_{i=1}^{5} D_{i}$.

Define $c_{0}=D_{0} \cup D_{1} \cup D_{3}, c_{1}=D_{2} \cup D_{4} \cup D_{5}$ $\bigcup\{0\}$, the generalized Hall's sextic sequence $\$ S \$$ is defined by



Define

$$
S(x)=S_{0}+S_{1} x+\cdots+s_{N-1} x^{N-1}=\sum_{i \in \Omega} x^{i}
$$

as the characteristic polynomial of the sequence $S$. It is well-known that the connection polynomial of the binary sequence $S$ of period $N$ is given by

$$
f_{s}(x)=\frac{1-x^{N}}{\operatorname{gcd}\left(1-x^{N}, S(x)\right)},
$$

where the constant coefficient of $\operatorname{gcd}\left(1-x^{N}, S(x)\right)$ is 1. So the linear complexity of $\$ S \$$ is given by

$$
L=N-\operatorname{deg}\left(\operatorname{gcd}\left(1-x^{N}, S(x)\right)\right) \text {. }
$$

In this paper, we will compute the connection polynomial and the linear complexity of the sequences (1.1).

\section{LINEAR COMPLEXITY}

Let $p=6 f+1$ be a prime. There is a decomposition over $Z: p=A^{2}+3 B^{2}, \quad A \equiv 1(\bmod 3)$. We define the sign of $B$ as follows: if $B \equiv 0(\bmod 3)$, then $B / 3$ $\equiv$ ind $_{\theta} 3(\bmod 3)$ and always $B \equiv-$ ind $_{\theta} 2(\bmod 3)$, where $i=\operatorname{ind}_{\theta} 2(\bmod 3)$ is the smallest positive integer such that $\theta^{i}=2(\bmod p)$.

We define the polynomial 


$$
S_{j}(x)=\sum_{i \in D_{j}} x^{i}, j=0,1,2,3,4,5 .
$$

Let $\alpha$ be a primitive $p_{\text {th root of unity in an extension }}$ of $\mathrm{GF}(2)$. Define

$S=\left(S_{0}(\alpha), S_{1}(\alpha), S_{2}(\alpha), S_{3}(\alpha), S_{4}(\alpha), S_{5}(\alpha)\right)$.

Let $p=A^{2}+3 B^{2}, A \equiv 1(\bmod 3)$. By [7], we have the following results.

ind $_{\theta} 2 \equiv 0(\bmod 3), \quad$ that is, $A=4+6 a, B=3+6 b$,then

(1) $S=(1,1,0,0,1,0) \quad$ if $\quad a \equiv 1(\bmod 2)$, $b \equiv 0(\bmod 2)$;

(2) $\quad S=(1,0,1,0,0,1) \quad$ if $\quad a \equiv 1(\bmod 2)$, $b \equiv 1(\bmod 2)$;

(3) $S=(\gamma, 0,1,1,-\gamma, 0,1)$ where $\gamma$ is a root of the equationif : and $b \equiv 1$

$(\bmod 2)$;

(4) $S=(\gamma, 1,0,1,-\gamma, 1,0)$ if and $b \equiv 1$

$(\bmod 2)$.

If $f \equiv 0(\bmod 2), A=1+6 a, B=6 b$, then

(5) $S=(1,0,0,0,0,0)$ if $b \equiv 0(\bmod 2)$;

(6) $S=(1,1,1,0,1,1)$ if $b \equiv 1(\bmod 2)$;

(7) $S=(\gamma, 0,0,1,-\gamma, 0,0)$ ifand

$b \equiv 1(\bmod 2)$;

(8) $S=(\gamma, 1,1,1,-\gamma, 1,1)$ if $a \equiv 1(\bmod 2)$, and

$b \equiv 1(\bmod 2)$.

Now we compute the linear complexity of the sequence defined as (1.1). Then by Blahut's theorem the linear comple $S$ xity of the sequence $S$ defined as (1.5) is

$L=p-\left|\left\{t \mid S\left(\alpha^{t}\right)=0, t=0,1, \cdots, p-1\right\}\right|$,

where $\quad S(x)=s_{0}+s_{1} x+\cdots+s_{p-1} x^{p-1} \quad$ is the characteristic polynomial of the sequence $S$. So the linear complexity of the sequence $S$ reduces to counting the number of roots of $S(x)$ in the set $\left\{\alpha^{t} \mid t=0,1, \cdots, p-1\right\}$.

It is clear that the characteristic polynomial of the sequence $S$ in (1.1) is $S(x)=S_{0}(x)+S_{1}(x)+S_{3}(x)$.

Now we compute the linear complexity $L$ and the connection polynomial $f_{S}(x)$ of the Hall's sextic cyclotomic sequence. Let $\alpha$ be a $p$-th primitive root of unity in an extension field over $\mathrm{GF}(2)$. Set

$$
d_{i}(x)=\prod_{l \in D_{i}}\left(1-\alpha^{-l} x\right), i=0,1,2,3,4,5 .
$$

Theorem 2.1. Let $p=6 f+1$ be a prime, $p=A^{2}+3 B^{2}$,

$$
A, B \in Z, A \equiv 1(\bmod 3) .
$$

if $A=4+6 a, B=3+6 b, a, b \in Z$.

(1)If $a \equiv 1(\bmod 2)$ and $b \equiv 0(\bmod 2)$, then

$$
f_{s}(x)=(1-x) d_{5}(x), L=(p-5) / 6 .
$$

(2)If $a \equiv 1(\bmod 2)$ and $b \equiv 1(\bmod 2)$, then

$$
\begin{gathered}
f_{S}(x)=(1-x) d_{0}(x) d_{1}(x) d_{3}(x) d_{4}(x) d_{5}(x), \\
L=(5 p+1) / 6 .
\end{gathered}
$$

(3)If $a \equiv 0(\bmod 2)$ and $b \equiv 0(\bmod 2)$, then

$$
f_{S}(x)=1-x^{p}, L=p .
$$

(4)If $a \equiv 1(\bmod 2)$ and $b \equiv 1(\bmod 2)$, then

$$
f_{S}(x)=(1-x) d_{2}(x) d_{5}(x), L=(p+2) / 3 \text {. }
$$

If $f=2, A=1+6 a, B=6 b, a, b \in Z$.

(5)If $a \equiv 0(\bmod 2)$ and $b \equiv 0(\bmod 2)$, then

$$
f_{S}(x)=d_{0}(x) d_{3}(x) d_{5}(x), L=(p-1) / 2 .
$$

(6)If $a \equiv 1(\bmod 2)$ and $b \equiv 1(\bmod 2)$, then

$$
f_{S}(x)=d_{1}(x) d_{4}(x) d_{5}(x), L=(p-1) / 2 .
$$

(7)If $a \equiv 0(\bmod 2)$ and $b \equiv 1(\bmod 2)$, then $f_{s}(x)=d_{0}(x) d_{2}(x) d_{3}(x) d_{5}(x), L=(2 p-2) / 3$.

(8)If $a \equiv 1(\bmod 2)$ and $b \equiv 1(\bmod 2)$, then $f_{S}(x)=d_{1}(x) d_{2}(x) d_{4}(x) d_{5}(x), L=(2 p-2) / 3$. Proof. If then it is clear that $S(1)=\frac{p-1}{2}=3 f=1(\bmod 2)$.

(1)If $a \equiv 1(\bmod 2)$ and $b \equiv 0(\bmod 2)$. If $a \in D_{i}$, then $S\left(\alpha^{i}\right)=S_{0}\left(\alpha^{i}\right)+S_{1}\left(\alpha^{i}\right)+S_{3}\left(\alpha^{i}\right)=S_{i}(\alpha)+S_{1+i}(\alpha)$ $+S_{3+i}(\alpha)$. Hence by Lemma 1 , we know that $S\left(\alpha^{i}\right)=0$ If $i \in D_{0} \cup D_{1} \cup D_{2} \cup D_{3} \cup D_{4}, \quad S\left(\alpha^{i}\right) \neq 0 \quad$ otherwise. Then $\operatorname{gcd}\left(1-x^{p}, S(x)\right)=d_{0}(x) d_{1}(x) d_{2}(x) d_{3}(x) d_{4}(x)$, so $f_{S}(x)=(1-x) d_{5}(x)$ and $L=(p-5) / 6$.

(2)If $a \equiv 1(\bmod 2)$ and $b \equiv 1(\bmod 2)$. Similarly, we have thatt $S\left(\alpha^{i}\right)=0$

If $i \in D_{2}, \quad S\left(\alpha^{i}\right) \neq 0 \quad$ otherwise. Then $\operatorname{gcd}\left(1-x^{p}, S(x)\right)=d_{2}(x)$, so

$$
f_{S}(x)=(1-x) d_{0}(x) d_{1}(x) d_{3}(x) d_{4}(x) d_{5}(x),
$$
and $L=(5 p+1) / 6$. 
(3)If $a \equiv 0(\bmod 2)$ and $b \equiv 0(\bmod 2)$. Similarly, we have that $S\left(\alpha^{i}\right) \neq 0$ for any $i \in Z_{p}$, then

and $L=p$.

(4)If $a \equiv 0(\bmod 2)$ and $b \equiv 1(\bmod 2)$. Similarly, we have that $S\left(\alpha^{i}\right)=0$ if $i \in D_{0} \cup D_{1} \cup D_{2} \cup D_{3} \cup D_{4}$,

$$
S\left(\alpha^{i}\right) \neq 0 \quad \text { otherwise. Then }
$$

$f_{S}(x)=(1-x) d_{2}(x) d_{5}(x)$,

and $L=(p+2) / 3$.

If $f \equiv 0(\bmod 2)$, then it is clear that $S(1)=\frac{p-1}{2}$

$=0(\bmod 2)$.

(5)If $a \equiv 0(\bmod 2)$ and $b \equiv 0(\bmod 2)$. Similarly, we have that $S\left(\alpha^{i}\right)=0$ if $i \in D_{1} \cup D_{2} \cup D_{4} \cup\{0\}$,

$$
S\left(\alpha^{i}\right) \neq 0 \quad \text { otherwise. }
$$

Then

$f_{S}(x)=d_{0}(x) d_{3}(x) d_{5}(x)$,

and $L=(p-1) / 2$.

(6)If $a \equiv 1(\bmod 2)$ and $b \equiv 0(\bmod 2)$. Similarly, we have that $S\left(\alpha^{i}\right)=0$ if $i \in D_{0} \cup D_{2} \cup D_{3} \cup\{0\}$,

$S\left(\alpha^{i}\right) \neq 0$ otherwise.

Then

$f_{S}(x)=d_{1}(x) d_{4}(x) d_{5}(x)$,

and $L=(p-1) / 2$.

(7)If $a \equiv 0(\bmod 2)$ and $b \equiv 1(\bmod 2)$. Similarly, we have that $S\left(\alpha^{i}\right)=0$ if $i \in D_{1} \cup D_{4} \cup\{0\}, S\left(\alpha^{i}\right) \neq 0$

otherwise. Then $f_{S}(x)=d_{0}(x) d_{2}(x) d_{3}(x) d_{5}(x)$, and $L=(2 p-2) / 3$.
(8)If $a \equiv 1(\bmod 2)$ and $b \equiv 1(\bmod 2)$. Similarly, we have that $S\left(\alpha^{i}\right)=0$ if $i \in D_{0} \cup D_{3} \cup\{0\}, S\left(\alpha^{i}\right) \neq 0$ otherwise. Then $f_{S}(x)=d_{1}(x) d_{2}(x) d_{4}(x) d_{5}(x)$, and $L=(2 p-2) / 3$.

\section{REFERENCES}

[1] J. Cao and Q. Yue,A nearly equidistance cyclotomic sequence of order 6 over GF(3), Journal of Xuzhou Normal University (Natural Science Edition), Vol. 29 (3), 9-13, Sep. 2011.

[2] C. Ding, Linear complexity of generalized cyclotomic binary sequences of order 2, Finite Fields Appl., vol. 3, pp. 159-174, July 1997.

[3]C. Ding, Autocorrelation values of generalized cyclotomic sequences of order two, IEEE Trans. Inform. Theory, vol. 44, pp. 1698-1702, July 1998.

[4] V. A. Edemskii, On the linear complexity of binary sequences on the basis of biquadratic and sextic residue classes, Discrete Math. Appl., Vol.20, No.1,pp.75-84, 2010.

[5]V. A. Edemskii, About computation of the linear complexity of generalized cyclotomic sequences with period $p^{n+1}$, to appear Des. Codes Cryptogr., V. 61, No 3, 251-260, 2011.

[6] L. Hu, Q. Yue and M. Wang, The linear complexity of Whiteman's generalized cyclotomic sequences of period $p^{m+1} q^{n+1}$, IEEE Tran. Inform. Theory, V. 58, 5534-5543, 2012.

[7] J. H. Kim, H. Y. Song, On the linear complexity of Hall's sextic residue sequences, IEEE Trans. Inform. Theory 47, 2094-2096, 2001.

[8] M. Lei and Q. Yue, The Autocorrelation values of several cyclotomic sequences over $\mathrm{GF}(3)$, Computer engineering and science, V.30, No. 10, 30-32, 2011.

[9] A. Lempel, M. Cohn and W. L. Estman, A class of binary sequences with optimal autocorrelation properties, IEEE Trans. Inform. Theory 23, pp. 38-42, Jan. 1997.

[10] Fang Liu, Daiyuan Peng, Xiaohu Tang and Xianhua Niu, On the autocorrelation and the linear complexity of q-ary prime n-square sequences, Sequences and Their Applications- SETA 2010, Lecture Notes in Computer Science, 2010, Volume 6338/2010, 139-150,

[11]V. M. Sidelnikov, Some k-valued pseudo-random sequences and nearly equidistance codes, Problem of Information Transmission 5, 12-16, 1969. 